DOI: https://doi.org/10.15688/mpcm.jvolsu.2019.2.7

UDC 541.14: 544.522

Submitted: 07.12.2018

LBC 24.5

Accepted: 04.03.2019

\title{
EFFECT OF VIBRATIONAL TRANSITIONS ON THE NONTHERMAL CHARGE TRANSFER PROBABILITY ${ }^{1}$
}

\author{
Elena Alexandrovna Mikhailova \\ Candidate of Physical and Mathematical Sciences, Associate Professor, \\ Department of Theoretical Physics and Wave Processes, \\ Volgograd State University \\ mixailova@volsu.ru, tf@volsu.ru \\ Prosp. Universitetsky, 100, 400062 Volgograd, Russian Federation
}

\section{Valentina Alexandrovna Mikhailova}

Doctor of Physical and Mathematical Sciences,

Head of Department of Theoretical Physics and Wave Processes, Volgograd State University mikhailova.va@volsu.ru,tf@volsu.ru https://orcid.org/0000-0002-9123-0391

Prosp. Universitetsky, 100, 400062 Volgograd, Russian Federation

\begin{abstract}
In the framework of the stochastic approach, the effect of transitions between the vibrational sublevels of products on the ultrafast dynamics of "hot" (non-thermal) electron transfer in donor - acceptor complexes dissolved in a polar medium has been investigated. An analytical expression has been obtained for the probability of electron transfer, which takes into account the transition between the vibrational states of products and the mutual influence of sinks. Quantitative estimates of the scale of the mutual influence of sinks on the probability of electron transfer are made.
\end{abstract}

Key words: charge recombination, photoinduced reaction, charge transfer, intramolecular vibrational relaxation, nonequilibrium of nuclear system.

\section{Introduction}

Charge transfer $(\mathrm{CT})$ reaction has been extensively studied for several decades $[4 ; 7 ; 13]$. Despite the significant progress in research of the electron transfer (ET) reaction by both experimental and theoretical methods, there are still problems in understanding its detailed mechanism and especially in controlling of its effectiveness. One of the reasons is the large 
number of regimes in which ET can proceed and as well as the variety of energical parameters controlling this process. In different regimes, the ET rate constant dependencies on the controlling parameters can vary greatly, and in some regimes they may even be opposite [4]. It creates difficulties in controlling ET in real molecular systems. To develop a purposeful search for ways to monitoring ET, it is necessary to know in which regime it proceeds and understand its mechanism.

The stochastic approach to the description of the kinetics of separation and transfer of charge in donor-acceptor systems in polar media, proposed in the early 80s [15-17], made it possible to consider different regimes of ET reactions, namely, nonadiabatic and solvent-controlled, within a single model as well as the transition between them. This approach consistently takes into account the formation of an energy barrier separating the states of reagents and products associated with the reorganization of the solvent [8;9]. It was shown that for a sufficiently large value of the electron coupling parameter $V_{e l}$, the reaction rate constant does not depend on it and is controlled only by the dynamics of the solvent $[16 ; 17]$ This regime in the current literature is called solution-controlled mode.

Another important generalization of the Zusman stochastic model was the consideration of the reorganization of intramolecular high-frequency oscillations excited in reagents during ET. In a number of works, it was shown that the rapid decay of reactant and products, associated with the relaxation of excited vibrational states, can significantly accelerate the ET reaction $[1 ; 3 ; 5 ; 10 ; 11 ; 14]$.

In this work, in the framework of the stochastic approach, we study the effect of transitions between vibrational sublevels of products on the ultrafast dynamics of hot (non-thermal) electron transfer in donor-acceptor complexes (DACs) dissolved in a polar environment Using the quasistationary approximation we drive an analytical expression for the probability of non-equilibrium charge transfer taking account a transition between the vibrational states of products and the mutual influence of the ET-sinks and estimate the effect of the vibrational transition on the kinetics of charge transfer occuring non-equilibrium regime.

\section{Stochastic point-transition model}

For a quantitative description of the kinetics of back charge transfer (charge recombination) in donor-acceptor complexes after their photoexcitation at the CT absorption band frequency, the three states of system "DAC+ polar solvent" are considered: the state with separated charges $D^{+} A^{-}$formed by photoexcitation of DACs, and two neutral states, one of which is vibrationally excited state of the complex in the ground electronic state (the intermediate product state), and the other is the state without vibrational excitation (the final product). It is assumed that the charge separated state $D^{+} A^{-}$is initially populated, while the last two states are populated as a result of electronic and vibrational transitions proceeding in parallel with the solvent relaxation (see figure 1). The effect of solvent polarization on the CR kinetics is described in the framework of the Debye model with the single relaxation time-scale. In this case the relaxation properties of a solvent is characterised by the autocorrelation function $X(t)=\exp \left(-t / \tau_{L}\right)$, where $\tau_{L}$ is longitudinal solvent relaxation time $[15 ; 17]$. Within the model the solvent relaxation mode corresponds to a reaction coordinate $Q$ and Gibbs free energies for the considered states are selected as $[6 ; 10]$ :

$$
U_{1}=\frac{Q^{2}}{4 E_{r m}}, U_{2}^{(j)}=\frac{\left(Q-Q_{m i n}\right)^{2}}{4 E_{r m}}+\Delta G_{0}+j \hbar \Omega, \quad j=0,1 .
$$


Here $E_{r m}$ and $-\Delta G_{0}$ are solvent reorganization energy and "driving force" of ET reaction (the free energy gap of ET), respectively. The intramolecular vibration of DACs is further considered to be the high-frequency one, the frequency of which $\Omega$ satisfies the condition $\hbar \Omega>>k_{B} T$, which reflects the quantum character of this mode. Here $k_{B}$ is the Boltzmann constant, $T$ is the temperature. Further, only two vibrational states of the product are considered: the ground $(j=0)$ and the first excited $(j=1)$ states. In figure 1 vibrationally excited state term is shown by a dashed line. The transitions between the electronic states are modeled by the $\delta$-shaped sinks localized at the term intersection points $Q_{0}^{*}$ and $Q_{1}^{*}$ (see fig. 1). The power of $j$ th sink $(j=0,1)$ depends on the electronic matrix element $V_{j}$ and equals to $2 \pi V_{j}^{2} / \hbar$, where $\hbar$ is the Planck constant.

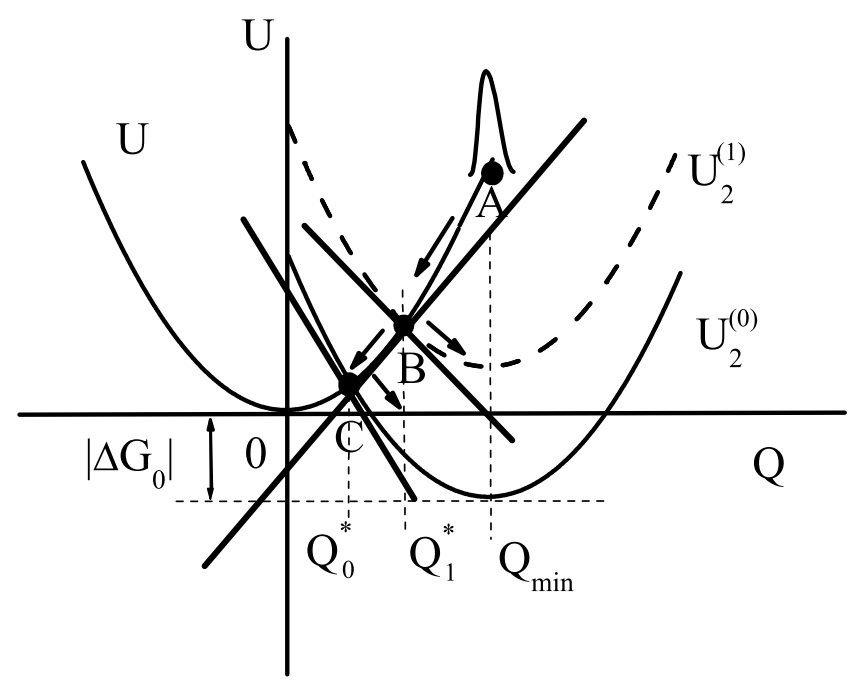

Fig. 1. A schematic representation of the free energy along the reaction coordinate $Q$ for the two electronic states of the complex $D^{+} A^{-}$(reactant, $U_{1}$ ) and $D A$ (product, $U_{2}^{(0)}$ ). The solid lines show the terms of the ground vibrational state of reactants and products, the dashed one is the first vibrationally excited state of the product $U_{2}^{(1)}$. Solvent relaxation is schematically indicated by arrows. The initial nonequilibrium state of the system represents the wave packet

The temporal evolution of the considered system "DAC + polar solvents" within the stochastic point-transition model is described by the set of Smoluchowski equations for the probability distribution functions of the reactant state $\rho_{1}$, the ground $\rho_{2}^{(0)}$ and the first vibrationally excited $\rho_{2}^{(0)}$ states of products, respectively $[1 ; 5 ; 6 ; 10 ; 13 ; 15 ; 17]$

$$
\begin{array}{r}
\frac{\partial \rho_{1}(Q, t)}{\partial t}=L_{1} \rho_{1}-\frac{2 \pi}{\hbar} \sum_{j=0}^{n=1} V_{j}^{2}\left(\rho_{1}-\rho_{2}^{(j)}\right) \delta\left(Q-Q_{j}^{*}\right), \\
\frac{\partial \rho_{2}^{(0)}(Q, t)}{\partial t}=L_{2} \rho_{2}^{(0)}+\frac{2 \pi V_{0}^{2}}{\hbar}\left(\rho_{1}-\rho_{2}^{(0)}\right) \delta\left(Q-Q_{0}^{*}\right)+\frac{\rho_{2}^{(1)}}{\tau_{v}} \\
\frac{\partial \rho_{2}^{(1)}(Q, t)}{\partial t}=L_{2} \rho_{2}^{(1)}+\frac{2 \pi V_{1}^{2}}{\hbar}\left(\rho_{1}-\rho_{2}^{(1)}\right) \delta\left(Q-Q_{1}^{*}\right)-\frac{\rho_{2}^{(1)}}{\tau_{v}} .
\end{array}
$$


Here the Smoluchowski operators $L_{\alpha}(\alpha=1,2)$ depict the diffusion on the free energy surfaces $U_{\alpha}$ :

$$
L_{\alpha}=\frac{2 E_{r m}}{\tau_{L}}\left(\frac{\partial^{2} U_{\alpha}}{\partial Q^{2}}+\frac{\partial U_{\alpha}}{\partial Q} \frac{\partial}{\partial Q}+k_{B} T \frac{\partial^{2}}{\partial Q^{2}}\right)
$$

The evolutionary equations (2)-(4) should be complemented by the initial conditions specifying the non-equilibrium CR kinetics

$$
\rho_{1}(Q, t=0)=\delta\left(Q-Q_{\text {min }}\right), \rho_{2}^{(0)}(Q, t=0)=\rho_{2}^{(1)}(Q, t=0)=0 .
$$

So, we assume that the initial wave packet on the reactant term $U_{1}$ (point $\mathrm{A}$ in figure 1 ), is well-localized and placed above the term intersection points $Q_{1}^{*}$ and $Q_{0}^{*}$. In next subsection we find analytical solutions of equations (2)-(4) with the initial condition (6) and the expression for $\mathrm{CR}$ probability by method based on the term linearization in the vicinity of their crossing.

\section{Quasistationary approximation}

In order to analytically calculate the probability of non-equilibrium $\mathrm{CR}$, as well as the population of the reaction reactant and products, we find the solutions of the equations (2)-(4) substituting the straight lines for the parabolic free energies $U_{\alpha}$. In this case the diffusion operators (5) will be written as: $\widetilde{L}_{1}=A_{1} \partial / \partial Q+B \partial^{2} / \partial Q^{2}$ and $\widetilde{L}_{2 j}=A_{2 j} \partial / \partial Q+$ $+B \partial^{2} / \partial Q^{2}(j=0,1)$, where the parameter $B=2 E_{r m} k_{B} T / \tau_{L}$ is related to the diffusion coefficient of particles over parabolic terms 1 , and the parameter $A_{2 j}=-Q_{j}^{*} / \tau_{L}$ depends on the slope of term $U_{2}^{(j)}$ at the intersection point $Q_{j}^{*}(j=0,1)$. Hereinafter, the reactant term slope is assumed to be the same for both sinks $A_{1}=\left(E_{r m}+\Delta G_{0}\right) / \tau_{L}$ while the product term slopes can be different. The set of the steady-state equations on linear intersecting terms with a source of particles ( $I$ is power of this $\delta$-shaped source), which is located above the intersection points $Q_{j}^{*}$ (in the figure 1 these are tangent lines in points $\mathrm{B}$ and $\mathrm{C}$ ) is written as follows $[6 ; 10]$

$$
\begin{array}{r}
I \delta\left(Q-Q_{\text {min }}\right)+\widetilde{L}_{1} \rho_{1}+\frac{2 \pi}{\hbar} \sum_{j=0}^{n=1} V_{j}^{2}\left(\rho_{1}-\rho_{2}^{(j)}\right) \delta\left(Q-Q_{j}^{*}\right)=0, \\
\widetilde{L}_{20} \rho_{2}^{(0)}+\frac{2 \pi V_{0}^{2}}{\hbar}\left(\rho_{1}-\rho_{2}^{(0)}\right) \delta\left(Q-Q_{0}^{*}\right)+\frac{\rho_{2}^{(1)}}{\tau_{v}}=0, \\
\widetilde{L}_{21} \rho_{2}^{(1)}+\frac{2 \pi V_{1}^{2}}{\hbar}\left(\rho_{1}-\rho_{2}^{(1)}\right) \delta\left(Q-Q_{1}^{*}\right)-\frac{\rho_{2}^{(1)}}{\tau_{v}}=0 .
\end{array}
$$

Applying Fourier transform

$$
\rho_{1}(Q)=\int_{-\infty}^{\infty} R_{1}(k) e^{i k Q} d k, \quad \rho_{2}^{(j)}(Q)=\int_{-\infty}^{\infty} R_{2 j}(k) e^{i k Q} d k
$$


we reduce the differential equation system (7)-(9) to an algebraic equation system

$$
\begin{array}{r}
\frac{I}{2 \pi} e^{-i k Q_{\min }}-\sum_{j=0}^{1} \frac{V_{j}^{2}}{\hbar}\left(\rho_{1}\left(Q_{j}^{*}\right)-\rho_{2}^{(j)}\left(Q_{j}^{*}\right)\right) e^{-i(k+i \varepsilon) Q_{j}^{*}}+i k A_{1} R_{1}-k^{2} B R_{1}=0, \\
\frac{V_{0}^{2}}{\hbar}\left(\rho_{1}\left(Q_{0}^{*}\right)-\rho_{2}^{(0)}\left(Q_{0}^{*}\right)\right) e^{-i(k+i \varepsilon) Q_{0}^{*}}+i k A_{20} R_{20}-k^{2} B R_{20}+\frac{R_{21}}{\tau_{v}}=0, \\
\frac{V_{1}^{2}}{\hbar}\left(\rho_{1}\left(Q_{1}^{*}\right)-\rho_{2}^{(1)}\left(Q_{1}^{*}\right)\right) e^{-i(k+i \varepsilon) Q_{1}^{*}}+i k A_{21} R_{21}-k^{2} B R_{21}-\frac{R_{21}}{\tau_{v}}=0
\end{array}
$$

and write the solutions:

$$
\begin{aligned}
& R_{1}=\sum_{j=0}^{1} \frac{V_{j}^{2}}{\hbar} \frac{\Delta \rho_{j}^{*} e^{-i(k+i \varepsilon) Q_{j}^{*}}}{k B\left(k-i\left|A_{1}\right| / B\right)}+\frac{I e^{-i k Q_{\min }}}{2 \pi k B\left(k-i\left|A_{1}\right| / B\right)} \\
& R_{20}=-\frac{1}{\hbar k B\left(k+i\left|A_{20}\right| / B\right)}\left\{V_{0}^{2} \Delta \rho_{0}^{*} e^{-i(k+i \varepsilon) Q_{0}^{*}}-\frac{V_{1}^{2} \Delta \rho_{1}^{*} e^{-i(k+i \varepsilon) Q_{1}^{*}}}{\tau_{V}\left[k B\left(k+i\left|A_{21}\right| / B\right)+1 / \tau_{V}\right]}\right\} \\
& R_{21}=-\frac{V_{1}^{2}}{\hbar} \frac{\Delta \rho_{1}^{*} e^{-i(k+i \varepsilon) Q_{1}^{*}}}{k B\left(k+i\left|A_{21}\right| / B\right)+1 / \tau_{V}} .
\end{aligned}
$$

The following notation is used here: $\Delta \rho_{j}^{*}=\rho_{2}^{(j)}\left(Q_{j}^{*}\right)-\rho_{1}\left(Q_{j}^{*}\right),(j=0,1)$.

Performing the inverse Fourier transform for functions (14)-(16), we find analytical solutions for the probability distribution functions of all the considered states

$$
\begin{aligned}
\rho_{1}(Q) & =\frac{I}{\left|A_{1}\right|}\left\{\theta\left(Q-Q_{\text {min }}\right) e^{-\frac{\left|A_{1}\right|}{B}\left(Q-Q_{\text {min }}\right)}+\theta\left(Q_{\text {min }}-Q\right)\right\}+ \\
& +\frac{2 \pi I}{\hbar\left|A_{1}\right|} \sum_{j=0}^{1} V_{j}^{*} \Delta \rho_{j}^{*}\left\{\theta\left(Q-Q_{j}^{*}\right) e^{-\frac{\left|A_{1}\right|}{B}\left(Q-Q_{j}^{*}\right)}+\theta\left(Q_{j}^{*}-Q\right)\right\} \\
\rho_{2}^{(1)}(Q) & =-\frac{2 \pi V_{1}^{2} \Delta \rho_{1}^{*}}{\hbar\left|A_{21}\right| f_{V}}\left\{\theta\left(Q-Q_{1}^{*}\right) e^{-\frac{\left|A_{21}\right|}{2 B}\left(f_{V}-1\right)\left(Q-Q_{1}^{*}\right)}+\right. \\
& \left.+\theta\left(Q_{1}^{*}-Q\right) e^{-\frac{\left|A_{21}\right|}{2 B}\left(f_{V}+1\right)\left(Q_{1}^{*}-Q\right)}\right\}, \\
\rho_{2}^{(0)}(Q) & =-\frac{2 \pi V_{0}^{2} \Delta \rho_{0}^{*}}{\hbar\left|A_{20}\right|}\left\{\theta\left(Q-Q_{0}^{*}\right) e^{-\frac{\left|A_{20}\right|}{B}\left(Q-Q_{0}^{*}\right)}+\theta\left(Q_{0}^{*}-Q\right)\right\}- \\
& -\frac{2 \pi V_{1}^{2} \Delta \rho_{1}^{*}}{\hbar\left|A_{20}\right|}\left\{1-\frac{4 B\left|A_{20}\right|}{\tau_{V} f_{V}\left|A_{21}\right|^{3}} \frac{e^{-\frac{\left|A_{21}\right|}{2 B}\left(f_{V}-1\right)\left(Q-Q_{1}^{*}\right)}}{\left(f_{V}-1\right)\left(f_{V}-1+2\left|A_{20}\right| /\left|A_{21}\right|\right)}\right\} \theta\left(Q-Q_{1}^{*}\right)- \\
& -\frac{2 \pi V_{1}^{2} \Delta \rho_{1}^{*}}{\hbar\left|A_{1}\right|} \gamma(Q) \theta\left(Q_{1}^{*}-Q\right),
\end{aligned}
$$

where the designations are entered: $\theta(Q)$ is the Heaviside step function and

$$
\begin{array}{r}
\gamma(Q)=\frac{4 B\left|A_{1}\right|}{\tau_{V}\left|A_{20}\right|^{3}}\left\{\frac{\exp \left[\frac{\left|A_{20}\right|}{B}\left(Q-Q_{1}^{*}\right)\right]}{\left(\left(1+f_{V}\right) \frac{\left|A_{21}\right|}{\left|A_{20}\right|}-2\right)\left(2+\left(f_{V}-1\right) \frac{\left|A_{21}\right|}{\left|A_{20}\right|}\right)}\right. \\
\left.-\left[\frac{\left|A_{20}\right|}{\left|A_{21}\right|}\right]^{3} \frac{\exp \left[\frac{A_{21} \mid\left(1+f_{v}\right)}{2 B}\left(Q-Q_{1}^{*}\right)\right]}{f_{V}\left(1+f_{V}\right)\left(1+f_{V}-2 \frac{\left|A_{20}\right|}{\left|A_{21}\right|}\right)}\right\}
\end{array}
$$


and vibrational relaxation factor $[5 ; 10 ; 11]$

$$
f_{V}=\sqrt{1+4 B /\left(\tau_{L} A_{21}^{2}\right)}
$$

Using equations (17)-(19), one can obtain a system of algebraic equations for the definitions $\Delta \rho_{0}^{*}$ and $\Delta \rho_{1}^{*}$ and, as a result, write an analytical expression for the CR probability proceeding in the nonequilibrium mode

$$
W_{E T}=\frac{2 \pi V_{0}^{2}\left|\Delta \rho_{0}^{*}\right|}{\hbar I}=W_{12}^{(0)} \frac{1-W_{12}^{(1)}\left(1+\delta\left(Q_{0}^{*}\right)\right)}{1-W_{12}^{(0)} W_{12}^{(1)} e^{-\frac{\Delta G_{01}^{*}}{k_{B} T}}\left(1+\delta\left(Q_{0}^{*}\right)\right)} .
$$

Here the probability of transition on the $\mathrm{j}$-th sink in the absence of a neighboring one is determined by the well-known expression $[2 ; 5 ; 6 ; 10 ; 11]$

$$
W_{12}^{(j)}=\frac{2 \pi V_{j}^{2}}{\hbar A_{1}\left(1+g_{12}^{(j)}\right)}, j=0,1
$$

depending on the parameters of the non-adiabatic transition

$$
g_{12}^{(0)}=\frac{2 \pi V_{0}^{2}}{\hbar}\left(\frac{1}{\left|A_{1}\right|}+\frac{1}{\left|A_{20}\right|}\right), \quad g_{12}^{(1)}=\frac{2 \pi V_{1}^{2}}{\hbar}\left(\frac{1}{\left|A_{1}\right|}+\frac{1}{\left|A_{21}\right| f_{V}}\right),
$$

which in turn depend not only on the term slopes, but also on the factor that takes into account the vibrationally excited state decay $f_{V}$. The influence of oscillatory transitions and the mutual influence of stocks in the framework of the model under consideration is also determined by the parameter $\gamma\left(Q_{0}^{*}\right)$.

It should be noted that formulas (22)-(20) are a generalization of the results obtained earlier [10]. It is also easy to show that, in the absence of a transition between product vibrational sublevels, the parameter $\gamma\left(Q_{0}^{*}\right)$ vanishes, and for the CR probability, a simplified expression is obtained [2]

$$
W_{E T}=W_{12}^{(0)} \frac{1-W_{12}^{(1)}}{\left(1-W_{12}^{(0)} W_{12}^{(1)} \exp \left(-\Delta G_{01}^{*} / k_{B} T\right)\right)},
$$

that determines the probability of transition on the lower sink (index 0 ) in the presence of upper sink (index 1). The parameter $\Delta G_{01}^{*}$ in the formula 25 determines the magnitude of the vertical energy gap between the sinks. It is easy to get from the last formula $W_{E T}=W_{12}^{(0)}$ if there is no upper stock $\left(W_{12}^{(1)}=0\right)$.

Analytical expressions (17)-(24) allow to quantify the scale of the influence of vibrational transitions on non-equilibrium $\mathrm{CR}$ in the donor-acceptor complexes after their photoexcitation.

\section{Results and discussion}

The results of a study of the nonequilibrium CR kinetics which are carried out in the framework of the Debye medium model of the with a relaxation time $\tau_{L}=1$ ps at room 
temperature $k_{B} T=0,025 \mathrm{eV}$ presented in figures $2-4$. The solvent reorganization energy in all calculations was assumed to be fixed at $E_{r m}=1 \mathrm{eV}$, that is characteristic of strong polar solvents like acetonitrile. The magnitude of the free energy gap $\Delta G_{0}$ is varied from -0.6 to $0.6 \mathrm{eV}$, which guarantees the ET proceeding at the non-equilibrium stage (both sinks are in the Marcus normal region). The matrix electronic transition elements $V_{j}$ are chosen in the range of up to $0.025 \mathrm{eV}$. Variable parameters of the model in the calculations are the decay time of the intermediate product $\tau_{v}$. Analysis of the results makes it possible to formulate several trends connected with the mutual influence of the ET-sinks, and the effect of transitions between the product vibrational states on the nonequilibrium ET kinetics.

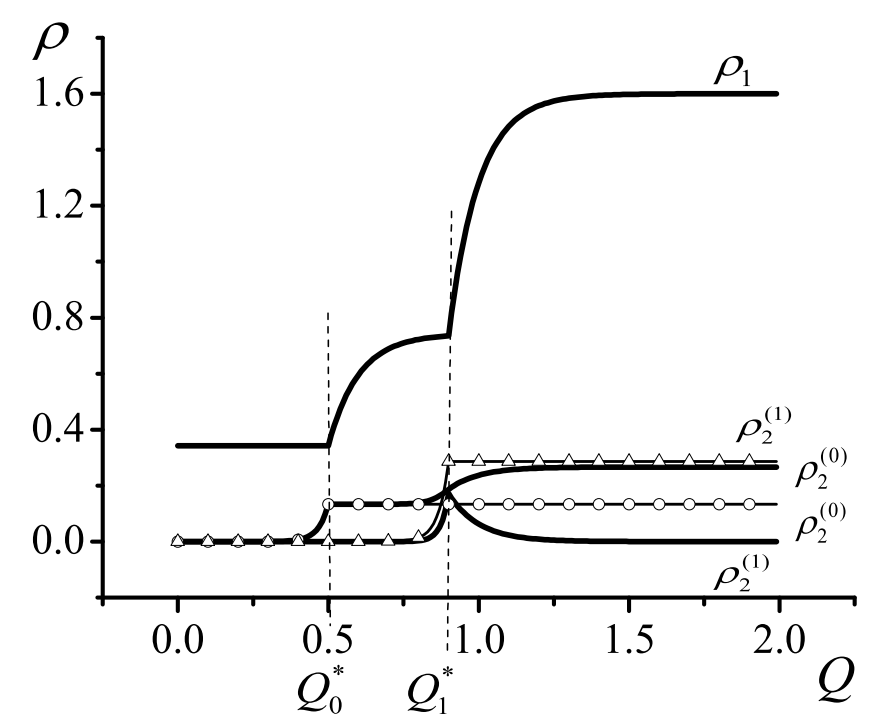

Fig. 2. Stationary probability distribution functions for system states $\rho_{1}, \rho_{2}^{(0)}$ and $\rho_{2}^{(1)}$ along the reaction coordinate $Q$. Calculated parameters are: $V_{0}=V_{1}=0.01 \mathrm{eV}, \Delta G_{0}=0, \Delta G_{01}^{*}>>k_{B} T$. The functions $\rho_{2}^{(0)}$ and $\rho_{2}^{(1)}$ are shown at following values of vibrational time-scale $\tau_{V}=50$ ps and $\infty$, as solid and dashed lines, respectively

First, as expected, due to the transition between the product vibrational sublevels, the function $\rho_{2}^{(0)}$ increases to the right of the sinks $\left(Q>Q_{1}^{*}>Q_{0}^{*}\right)$, while the function $\rho_{2}^{(1)}$ decreases to zero (compare solid and symbol lines in Figure 2). Secondly, the vibrational relaxation strongly affects on the ET probability $W_{E T}$ (as well as on the ET probability of a vibrationally excited state $W_{12}^{(1)}$ ). It is enough to compare the dashed (relaxation is absent) and symbol (fast relaxation) lines 2 and 1, 4 and 3 in Figure 3. Thirdly, the ET probability $W_{E T}$ is sensitive not only to the vibrational relaxation time-scale, but also to the distance between sinks. The vibrational relaxation effect is the strongest when the sinks coincide (lines 4 and 3 in Figure 3). The influence of the variation of distance between sinks from zero to $0.5 \mathrm{eV}$ on the ET probability is shown in Figure 4. When the transition between vibrational sublevels is absent $\left(\tau_{V} \rightarrow \infty\right)$ and the distance between sinks is equal to zero, the ET probabilities $W_{E T}=W_{12}^{(1)}$ (dashed line 4). The decay of the vibrationally excited state $\left(\tau_{V}=50\right.$ fs, symbol lines 3$)$ changes the probabilities: $W_{E T}$ decreases, and $W_{12}^{(1)}$ increases by the same value. The mutual influence of stocks in the area $\Delta Q_{01}^{*}>0.1 \mathrm{eV}$ is demonstrated by dashed lines 2 (Figure 3). 


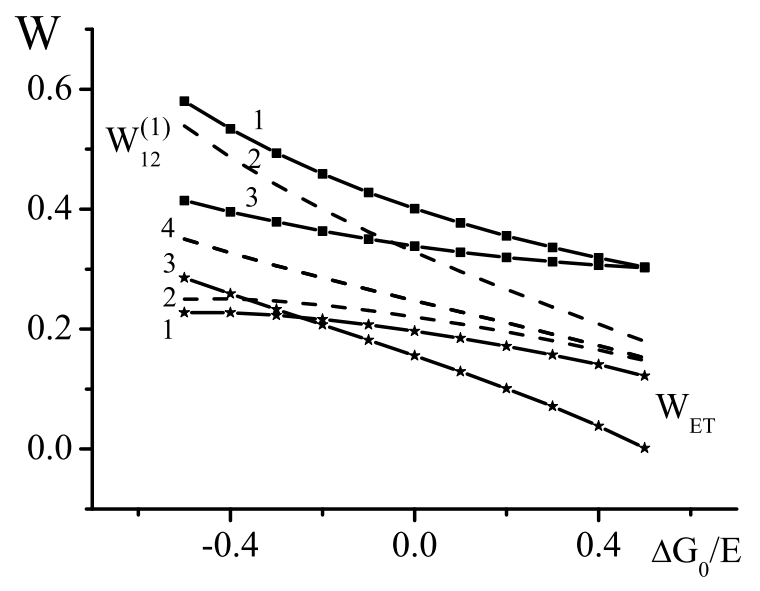

Fig. 3. Dependencies of the probabilities $W_{E T}$ and $W_{12}^{(1)}$ on the free-energy gap $\Delta G_{0}$. Calculated parameters are: $V_{0}=V_{1}=0.01 \mathrm{eV}$, (lines 1) $\tau_{V}=50 \mathrm{ps}, \Delta Q_{01}^{*}=0.4 \mathrm{eV}$; (lines 2) $\tau_{V} \rightarrow \infty$, $\Delta Q_{01}^{*}=0.4 \mathrm{eV}$; (lines 3) $\tau_{V}=50 \mathrm{ps}, \Delta Q_{01}^{*}=0$; (lines 4) $\tau_{V} \rightarrow \infty, \Delta Q_{01}^{*}=0$

It should be emphasized that in this work only transitions between the vibrational sublevels of the products are considered. However, there is a number of processes where a significant role is played transitions between the vibrational sublevels of the reactants. For example, in photoinduced electron transfer reactions, excited states of high-frequency vibrational modes often arise under the influence of a laser pulse, so that ultrafast charge separation occurs from vibrationally excited states. A study showed that the vibrational mode excitation can significantly affect the ET kinetics $[11 ; 12]$.

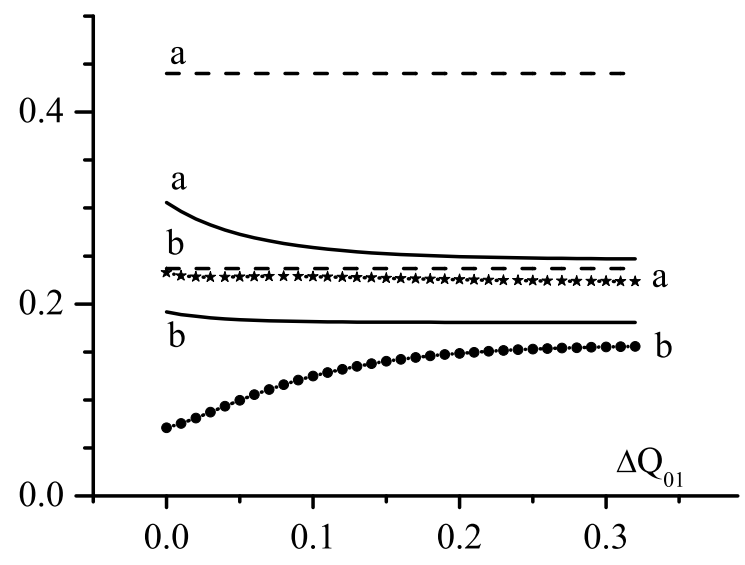

Fig. 4. Dependencies of the probabilities $W_{E T}$ on the distance between $\delta$-shaped sinks $\Delta Q_{01}^{*}$. Calculated parameters are: $V_{0}=V_{1}=0.01 \mathrm{eV} ; \Delta G_{0}=-0.3 \mathrm{eV}$ (line series a) and $0.3 \mathrm{eV}$ (line series b); $\tau_{V}=50$ ps (symbol lines) and $\tau_{V} \rightarrow \infty$ (solid lines). Dashed lines show the probability of transition on the 0 -th sink in the absence of a neighboring one

Thus in this paper, within of the stochastic point-transition model, the effect of transitions between the vibrational states of products and the mutual influence of two ET sinks 
on the of non-equilibrium charge transfer probability is explored. Analytical expressions obtained for CT probability and the probability distribution functions allow to quantify the influence scale of the vibrational transition on ET kinetics. It is shown that due to the mutual influence of sinks and vibrational relaxation, the probability of charge transfer at the nonequilibrium stage can change significantly.

\section{REMARK}

${ }^{1}$ The study was performed by a grant 16-13-10122 from the Russian Science Foundation.

\section{REFERENCES}

1. Feskov S.V., Ionkin V.N., Ivanov A.I. Effect of High-Frequency Modes and Hot Transitions on Free Energy Gap Dependence of Charge Recombination. J. Phys. Chem. A, 2006, vol. 110, no. 43, pp. 11919-11925. DOI: 10.1021/jp063280z.

2. Feskov S.V., Ivanov A.I. Efficiency of Intramolecular Charge Separation from the Second Excited State: Suppression of the Hot Charge Recombination by Electron Transfer to the Secondary Acceptor. J. Phys. Chem. A, 2013, vol. 117, pp. 11479-11489. DOI: $10.1021 / \mathrm{jp} 408516 \mathrm{q}$.

3. Ivanov A.I., Ionkin V.N., Feskov S.V. Acceleration of the Recombination of Photoexcited Donor-Acceptor Complexes with a High-Frequency Vibrational Mode. Russ. J. Phys. Chem. A, 2008, vol. 82, no. 2, pp. 303-309. DOI: 10.1134/S0036024408020295.

4. Feskov S.V., Mikhailova V.A., Ivanov A.I. Non-Equilibrium Effects in Ultrafast Photoinduced Charge Transfer Kinetics. J. Photochem. Photobiol. C, 2016, vol. 29, no. 12, pp. 48-72. DOI: 10.1016/j.jphotochemrev.2016.11.001.

5. Ivanov A.I., Mikhailova V.A. The Influence of Product Decomposition on the Probability of Nonthermal Transitions in Charge Transfer Reactions. Russ. J. Phys. Chem. B, 2008, vol. 2, no. 5, pp. 663-670. DOI: 10.1134/S1990793108050011.

6. Ivanov A.I., Potovoi V.V. Theory of non-thermal electron transfer. Chemical Physics, 1999, vol. 247, no. 2, pp. 245-259. DOI: 10.1016/S0301-0104(99)00197-4.

7. Kumpulainen T., Lang B., Rosspeintner A., Vauthey E. Ultrafast Elementary Photochemical Processes of Organic Molecules in Liquid Solution. Chem. Rev., 2017, vol. 117, no. 16, pp. 10826-10939. DOI: 10.1021/acs.chemrev.6b00491.

8. Marcus R.A., Sutin N. Electron Transfers in Chemistry and Biology. Biochim. Biophys. Acta, 1985, vol. 811, no. 3, pp. 265. DOI: 10.1016/0304-4173(85)90014-X.

9. Marcus R.A. On the theory of oxidation-reduction reactions involving electron transfer. J. Chem. Phys., 1956, vol. 24, no. 5, pp. 966-978. DOI: 10.1063/1.1742723.

10. Mikhailova V.A., Ivanov A.I. Effect of Relaxation of Intramolecular High Frequency Vibrational Mode on Nonthermal Electron Transfer Probability. Stochastic PointTransition Approach. J. Phys. Chem. C, 2007, vol. 111, no. 11, pp. 4445-4451. DOI: 10.1021/jp0678251.

11. Mikhailova V.A., Ivanov A.I. Effect of Reactant and Product State Decay on Ultrafast Charge-Transfer Kinetics: Violation of the Principle of Independence of Elementary Chemical Reactions. J. Phys. Chem. C, 2017, vol. 121, no. 17, pp. 20629-20639. DOI: 10.1021/acs.jpcc.7b06106.

12. Nazarov A.E., Barykov V.Yu., Ivanov A.I. Effect of Intramolecular High Frequency Vibrational Mode Excitation on Ultrafast Photoinduced Charge Transfer and Charge Recombination Kinetics. J. Phys. Chem. B, 2016, vol. 120, no. 12, pp. 3196-3205. DOI: 10.1021/acs.jpcb.6b00539.

13. Ivanov A.I., Mikhailova V.A. Kinetics of Fast Photochemical Charge Separation and Charge Recombination Reactions. Russ. Chem. Rev., 2009, vol. 79, no. 12, pp. 1047-1070. DOI: 10.1070/RC2010v079n12ABEH004167. 
14. Ulstrup J., Jortner J. The effect of intramolecular quantum modes on free energy relationships for electron transfer reactions. J. Chem. Phys., 1975, vol. 63, no. 10, pp. 4358. DOI: $10.1063 / 1.431152$.

15. Yakobson B.I., Burshtein A.I. Relaxation Hindrance in Nonadiabatic Cage Reactions. Chem. Phys., 1980, vol. 49, no. 385-395, pp. 3. DOI: 10.1016/0301-0104(80)850579 .

16. Zusman L.D. The dynamic effects of the solvent in electron transfer reactions. Russ. Chem. Rev., 1992, vol. 61, no. 15-24, pp. 1. DOI: 10.1070/RC1992v061n01ABEH000963.

17. Zusman L.D. Outer-Sphere Electron Transfer in Polar Solvents. , 1980, vol. 49, pp. 2955. DOI: $10.1016 / 0301-0104(80) 85267-0$.

\section{ВЛИЯНИЕ КОЛЕБАТЕЛЬНЫХ ПЕРЕХОДОВ НА ВЕРОЯТНОСТЬ НЕТЕРМИЧЕСКОГО ПЕРЕНОСА ЭЛЕКТРОНА}

\section{Елена Александровна Михайлова}

Кандидат физико-математических наук, доцент кафедры теоретической физики и волновых процессов, Волгоградский государственный университет mixailova@volsu.ru, tf@volsu.ru просп. Университетский, 100, 400062 г. Волгоград, Российская Федерация

\section{Валентина Александровна Михайлова}

Доктор физико-математических наук, заведующая кафедрой теоретической физики и волновых процессов, Волгоградский государственный университет mikhailova.va@volsu.ru, tf@volsu.ru https://orcid.org/0000-0002-9123-0391 просп. Университетский, 100, 400062 г. Волгоград, Российская Федерация

Аннотация. В рамках стохастического подхода исследовано влияние переходов между колебательными подуровнями продуктов на сверхбыструю динамику «горячего» (нетермического) переноса электрона в донорно-акцепторных комплексах, растворенных в полярной среде. Для вероятности переноса электрона получено аналитическое выражение, учитывающее переход между колебательными состояниями продуктов и взаимное влияние стоков. Сделаны количественные оценки масштаба взаимного влияния стоков на вероятность переноса электрона.

Ключевые слова: рекомбинация зарядов, фотоиндуцированные реакции, перенос заряда, внутримолекулярная колебательная релаксация, неравновесность ядерной системы. 